\title{
Predictors and outcomes of mycobacteremia among HIV-infected smear- negative presumptive tuberculosis patients in Uganda
}

Lydia Nakiyingi ${ }^{1,2^{*}}$, Willy Ssengooba ${ }^{2,3}$, Damalie Nakanjako ${ }^{1,2}$, Derek Armstrong ${ }^{4}$, Molly Holshouser ${ }^{4}$, Bruce J Kirenga ${ }^{2}$, Maunank Shah ${ }^{4}$, Harriet Mayanja-Kizza ${ }^{2}$, Moses L Joloba², Jerrold J Ellner ${ }^{5}$, Susan E Dorman ${ }^{4}$ and Yukari C Manabe $e^{1,4}$

\begin{abstract}
Background: Sputum smear microscopy for tuberculosis (TB) diagnosis lacks sensitivity in HIV-infected symptomatic patients and increases the likelihood that mycobacterial infections particularly disseminated TB will be missed; delays in diagnosis can be fatal. Given the duration for MTB growth in blood culture, clinical predictors of MTB bacteremia may improve early diagnosis of mycobacteremia. We describe the predictors and mortality outcome of mycobacteremia among HIV-infected sputum smear-negative presumptive TB patients in a high prevalence HIV/TB setting.

Methods: Between January and November 2011, all consenting HIV-infected adults suspected to have TB (presumptive TB) were consecutively enrolled. Diagnostic assessment included sputum smear microscopy, urine Determine TB lipoarabinomannan (LAM) antigen test, mycobacterial sputum and blood cultures, chest X-ray, and CD4 cell counts in addition to clinical and socio-demographic data. Patients were followed for 12 months post-enrolment.

Results: Of 394 sputum smear-negative participants [female, 63.7\%; median age (IQR) 32 (28-39) years], 41/394 (10.4\%) had positive mycobacterial blood cultures (mycobacteremia); all isolates were M. tuberculosis (MTB). The median CD4 cell count was significantly lower among patients with mycobacteremia when compared with those without (CD4 31 versus 122 cells/ $\mu \mathrm{L}, \mathrm{p}<0.001$ ). In a multivariate analysis, male gender [OR 3.4, 95\%Cl (1.4-7.6), $p=0.005$ ], CD4 count $<100$ cells/ $\mu \mathrm{L}$ [OR $3.1,95 \% \mathrm{Cl}(1.1-8.6), p=0.030$ ] and a positive lateral flow urine TB LAM antigen test [OR 15.3, 95\%Cl (5.7-41.1), p < 0.001] were significantly associated with mycobacteremia. At 12 months of follow-up, a trend towards increased mortality was observed in patients that were MTB blood culture positive (35.3\%) compared with those that were MTB blood culture negative (23.3\%) $(p=0.065)$.

Conclusions: Mycobacteremia occurred in 10\% of smear-negative patients and was associated with higher mortality compared with smear-negative patients without mycobacteremia. Advanced HIV disease (CD4 $\left.<100 \mathrm{cells} / \mathrm{mm}^{3}\right)$, male gender and positive lateral flow urine TB LAM test predicted mycobacteremia in HIV-infected smear-negative presumptive TB patients in this high prevalence TB/HIV setting.
\end{abstract}

Keywords: Predictors, Mortality, Mycobacterial infections, Bacteremia, Smear- negative, HIV, LAM, Sub-Saharan Africa

\footnotetext{
* Correspondence: lydikiyingi@yahoo.com

${ }^{1}$ Infectious Diseases Institute, Makerere University College of Health Sciences, Kampala, Uganda

${ }^{2}$ Makerere University College of Heath Sciences, Kampala, Uganda

Full list of author information is available at the end of the article
} 


\section{Background}

Tuberculosis (TB) incidence and mortality have increased dramatically because of the human immunodeficiency virus (HIV) epidemic. TB is the leading cause of death among HIV-infected persons in sub-Saharan Africa (SSA) [1-4]. Sputum smear microscopy for acid-fast bacilli (AFB), the most widely used TB diagnostic method, detects less than half of HIV-infected TB cases [5,6]. Sputum smear-negative pulmonary TB $[7,8]$ is associated with delayed diagnosis and treatment with subsequently high mortality [9-11] especially in TB-HIV co-infected patients. Mortality in sputum smear-negative HIV-infected patients is likely due in part to undiagnosed mycobacterial disease including mycobacteremia, an often fatal form of disseminated TB.

The prevalence of mycobacteremia among HIV-infected hospitalized febrile patients ranges from 9 to $22 \%$ [12-15]. In Uganda, mycobacteremia has been reported in $23 \%$ of hospitalized HIV-infected patients with sepsis, half of whom die within a month of admission [16]. Mortality in patients with mycobacteremia often results from delayed or missed TB diagnosis. Early diagnosis and treatment therefore has the potential to reduce mycobacteremia attributable mortality [17]. However, diagnosis of mycobacteremia is often delayed due to atypical presentation, which makes clinical diagnosis more difficult [18-21]. The high cost of TB blood culture and the need for sophisticated laboratory infrastructure make the test unaffordable for routine patient care in high prevalence HIV/TB resource-limited settings [22]. In addition, TB blood culture has a minimum turnaround time of 3 weeks $[23,24]$ which further contributes to delayed diagnosis and subsequent morbidity and mortality. Death occurs before TB blood culture results in the majority of patients [14]. Given the duration required for MTB growth in blood culture, clinical predictors of MTB bacteremia and rapid diagnostic tests may improve early diagnosis of mycobacteremia particularly in settings with increased HIV /TB co infection. Mortality in HIV- infected, smear-negative TB patients could be reduced through early identification and treatment of patients who have MTB bacteremia.

We have previously shown that positive TB blood culture was associated with positive urine TB lipoarabinomannan (LAM) antigen test [25]. As a rapid point-of-care test, the urine TB LAM test, when used with other clinical factors, could contribute to early MTB bacteremia diagnosis and rapid initiation of anti-tuberculosis therapy. We report the predictors and outcomes of mycobacteremia among HIV-infected sputum smear-negative presumptive TB patients in a high prevalence HIV/TB setting.

\section{Ethical considerations}

This study was nested within a TB diagnostic study that was approved by the Scientific Review Board of the
Infectious Diseases Institute (IDI), the Institutional Review Boards of the Joint Clinical Research Centre, Kampala, Uganda, Johns Hopkins University, Baltimore, USA, and the Uganda National Council for Science and Technology. Participants provided informed consent.

\section{Methods}

\section{Study design and setting}

The analysis was performed on data collected from participants that were prospectively enrolled in a TB diagnostic study that assessed the accuracy of the lateral flow urine Determine TB LAM Ag test (henceforth called the 'TB LAM' test) for the diagnosis of TB among HIV-infected TB suspects [25]. Participants were recruited from the outpatient adult Infectious Diseases Institute (IDI) Clinic [26] and the inpatient wards of the Mulago National Referral Hospital, Kampala, Uganda between January 2011 and November 2011.

\section{Patients recruitment}

HIV-infected patients clinically suspected to have TB (presumptive TB patients) aged $\geq 18$ years were recruited. A patient was clinically suspected to have TB if he or she reported having cough, fever, night sweats, or weight loss. Patients were excluded if they had taken anti-TB medication for more than two days within 60 days prior to enrolment. At enrolment, participants were interviewed to obtain socio-demographic and medical information before study specific specimens were collected. The participants provided two spot sputum samples for direct fluorescence microscopy (FM) and Ziehl-Neelsen (ZN) microscopy; mycobacterial growth indicator tube (MGIT) and Lowenstein-Jensen (LJ) sputum cultures. Sputum induction using 7\% nebulized hypertonic saline was performed for participants who were unable to spontaneously expectorate sputum. Blood for mycobacterial blood cultures and CD4 cell count was collected from each participant using aseptic techniques. Species identification on all positive TB cultures was performed. Urine was collected for TB LAM testing. Chest radiographs were obtained for all male participants and nonpregnant women. Further details on patient recruitment were described elsewhere [25]. The analysis included participants who were AFB smear-negative on both $\mathrm{ZN}$ and FM microscopy and had TB blood culture results available.

\section{Laboratory procedures}

After collection, sputum specimens were kept in a cool box until they were transferred to a refrigerator upon receipt in the mycobacteriology (BSL-3) laboratory under Makerere University, Department of Medical Microbiology located within the Mulago Hospital complex. Smears were prepared from each unprocessed sputum specimen 
and stained using $\mathrm{ZN}$ and auramine-O methods. The remaining portions of the sputum specimens were decontaminated using $\mathrm{N}$-acetyl-L-cysteine- $\mathrm{NaOH}$ of which $0.5 \mathrm{ml}$ was used to inoculate MGIT culture using the MGIT 960 system (Becton and Dickinson, Franklin Lakes, NJ USA) and $0.1 \mathrm{ml}$ to inoculate LJ culture media. Cultures were incubated at $37^{\circ} \mathrm{C}$ for up to six weeks for MGIT and eight weeks for the LJ method.

MYCO/F LYTIC (Becton and Dickinson) tubes were used for mycobacterial blood cultures; blood inoculum volume was $3 \mathrm{ml}$ and incubated in Bactec 9120 machine (Becton and Dickinson, Franklin Lakes, NJ USA) for up to six weeks. Positive cultures were assessed for presence of AFB using ZN staining and light microscopy, and for M. tuberculosis (MTB) complex using an anti MPB64 antibody assay (Capilia TB-Neo, TAUNS Laboratories, Numazu, Japan). Mycobacteremia was defined as isolation of mycobacteria from the mycobacterial blood culture.

For the TB LAM test, $60 \mu \mathrm{l}$ was pipetted onto the sample pad. According to the manufacturer's instructions, the strip was read 25 minutes later by two different technicians independently who compared the test strips with the reference card provided by the manufacturer and graded the result from $1+$ to $5+$. A result was considered positive if the band was graded as $2+$ or above.

CD4 cell count was performed at a certified laboratory at the IDI [26] following the laboratory standard procedure.

All study TB laboratory results (except for the urine TB LAM test that was an investigational test) were made available to the attending clinicians. Discharged participants were contacted by telephone to deliver TB results and those whose $\mathrm{TB}$ tests were positive were requested to return for TB treatment. During the phone interviews, participants were also asked if TB treatment had been initiated. Participants whose TB results were positive but could not be contacted by telephone had study home visits performed during which, information on TB treatment and survival status was obtained. MTBpositive patients (sputum smear positive by any of the methods or sputum culture positive by any method or blood culture positive) were immediately initiated on TB treatment by the attending clinician according to the guidelines from the Uganda Ministry of Health TB and Leprosy program [27].

\section{Assessment of mortality}

Information on survival status was obtained during monthly phone interviews that were conducted for a period up to 12 months post- enrolment. For patients who died in the hospital, the date of death was recorded. For patients who were discharged, they or their family were contacted by mobile phone at least monthly after enrolment to obtain survival status. For those who died, the date of death was recorded; if the exact date of death was not available, the date of death was recorded as the date of the follow-up phone call.

\section{Data management and statistical analysis}

Smear-negative participants were primarily stratified according to their TB blood culture status reported as either positive or negative. Continuous variables were summarized using medians and inter-quartile ranges (IQR) while categorical variables were summarized using frequencies, proportions and percentages. Using Wilcoxon rank sum test for continuous variables and Chi-square test or Fisher's exact test for categorical variables, we compared the characteristics of the study population stratified by TB blood culture status.

To identify predictors of mycobacteremia among sputum smear-negative HIV-infected presumptive TB patients, a multivariate logistic regression model was constructed using all factors from bivariate analysis that had a p-value of $\leq 0.2$ and those of known clinical significance, specifically chest X-ray (CXR) findings. Using a systematic backward elimination approach, non-significant variables were removed from the model until no further variables were eligible for removal to arrive at the final parsimonious model. A p-value of $<0.05$ in the final model was considered statistically significant.

In the assessment of mortality, the endpoint was mortality 12 months post- enrolment. Kaplan-Meier estimates of mortality between participants with and without mycobacteremia were compared using the log-rank test.

All data were analyzed using STATA $^{\circ}$ version 12.0 (StataCorp, 4905 Lakeway Drive College Station, Texas USA).

\section{Results}

\section{Study participants' characteristics}

Of the 501 TB suspects who provided blood for TB blood culture testing, 394 were sputum smear-negative and eligible for the analysis (Figure 1); $63.7 \%$ were female with a median age (IQR) and CD4 cell count (IQR) of 32 (28-39) years and $106(24-308)$ cells $/ \mathrm{mm}^{3}$, respectively (Table 1$)$. Of the $68.5 \%$ (270/394) who were hospitalized, the median $\mathrm{CD} 4$ was lower compared to the non-hospitalized participants [59 cells/ $\mu \mathrm{L}$ (IQR 14-182) versus 281 cells/ $\mu \mathrm{L}$ (IQR 106-491), respectively, $\mathrm{p}<0.001$ ].

\section{Mycobacteremia in study population}

Of the 394 sputum smear-negative participants, 41/394 $(10.4 \%)$ had positive mycobacterial blood cultures, and all cultured isolates were M. tuberculosis (MTB) complex on species identification. The average time to positivity for the MTB positive blood cultures was $24( \pm 8 \mathrm{SD})$ days. All 41 participants with positive MTB blood 


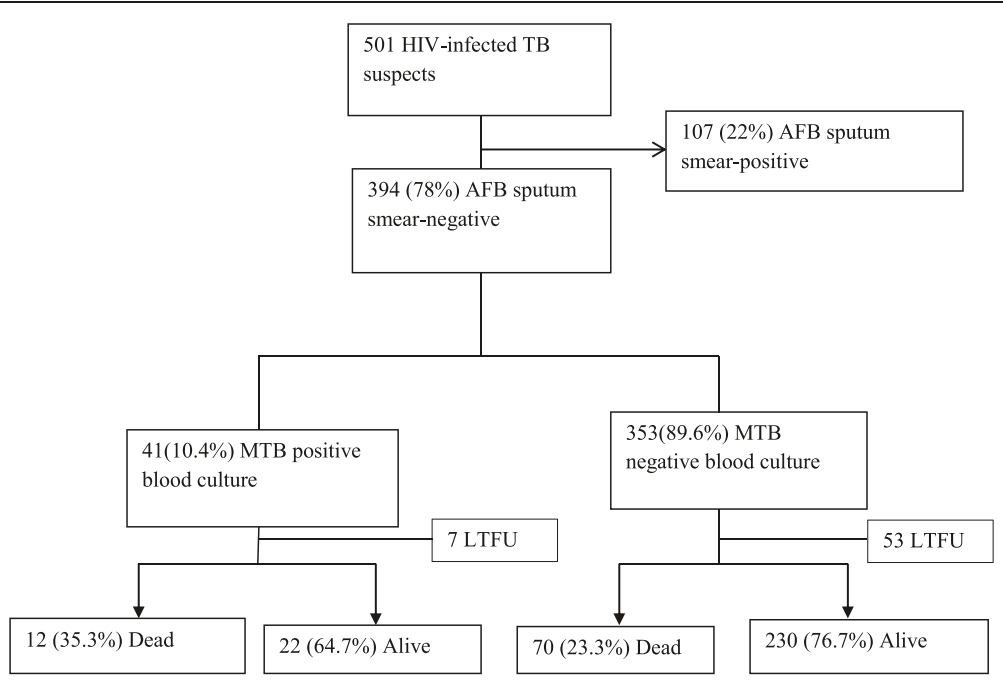

Abbreviations: AFB, Acid-fast bacilli; LTFU, Lost to follow-up

Figure 1 Participant flow diagram.

cultures reported excessive weight loss (i.e. weight loss of $>10 \%$ in four weeks) and the majority, 31/41(75.6\%) had one or more sputum cultures positive for MTB on LJ and/ or MGIT culture. Among the 10 MTB blood culture-positive, sputum culture-negative participants, all $(10 / 10,100 \%)$ were hospitalized, had a normal CXR and $5 / 10(50 \%)$ had CD4 cell count $<50$ cells $/ \mathrm{mm}^{3}$ [median CD4 $(\mathrm{IQR})=50(7-137)$ cells $\left./ \mathrm{mm}^{3}\right]$. Urine TB LAM test was positive in $70.7 \%$ (29/41) of the MTB blood culture-positive participants.

\section{Predictors of mycobacteremia in HIV-infected} smear-negative participants

Results of the bivariate analysis are summarized in Table 2. On multivariate logistic regression analysis, male gender [OR 3.4, 95\%CI (1.4-7.9), $\mathrm{p}=0.005$ ], CD4 cell count $<100$ cells $/ \mu \mathrm{L}$ [OR 3.1, 95\%CI (1.1-8.6), $\mathrm{p}=$
0.030] and a positive urine TB LAM test [OR 15.3, 95\% CI (5.7-41.1), $\mathrm{p}<0.001]$ were independently associated with mycobacteremia in HIV-infected sputum smearnegative clinically suspected TB patients.

\section{Mortality in participants with mycobacteremia}

Follow-up information was available for 34/41 (82.9\%) of the sputum smear-negative participants with positive MTB blood culture (mycobacteremia) and 300/353 (85\%) of the blood culture-negative participants. The remaining participants (7 with mycobacteremia and 53 without mycobacteremia) could not be reached by phone or home visits.

At 12 months, a trend towards increased mortality existed in those that were MTB blood culture positive (35.3\%, 12/34) compared to those that were MTB blood culture negative $(23.3 \%, 70 / 300)$ although this difference

Table 1 Comparing characteristics of sputum smear-negative HIV-infected presumptive TB patients with and without mycobacteremia, $\mathrm{N}=394$

\begin{tabular}{|c|c|c|c|c|}
\hline Characteristics & Total, $\mathrm{N}=394$ & Positive TB blood culture $\mathrm{N}=41$ & Negative TB blood culture $\mathrm{N}=353$ & $P$ value \\
\hline Median age $(\mathrm{QQR})$ years & $32(28-39)$ & $35(28-40)$ & $32(27-39)$ & 0.260 \\
\hline Male, \# (\%) & $143(36.3)$ & $24(58.5)$ & 119(33.7) & 0.002 \\
\hline Hospitalization & $270(68.5)$ & $36(87.8)$ & $234(66.3)$ & 0.005 \\
\hline Median CD4 cells $/ \mathrm{mm}^{3}$ (IQR) & $106(24-308)$ & $31(10-73)$ & $122(28-323)$ & $<0.001$ \\
\hline CD4 $<100$ & $190(48.2)$ & $35(85.4)$ & $155(43.9)$ & $<0.001$ \\
\hline Not on ART, \# (\%) & $246(62.4)$ & $31(75.6)$ & 215(60.9) & 0.070 \\
\hline${ }^{* *}$ CXR abnormal, \# (\%),N = 361* & 209(57.9\%) & $21(61.8 \%)$ & $188(57.5 \%)$ & 0.631 \\
\hline Sputum culture positive (MGIT/or LJ) & $88(22.3)$ & $31(75.6)$ & $57(16.1)$ & $<0.001$ \\
\hline *Positive urine TB LAM test $(\mathrm{N}=393)$ & $52(13.2)$ & $29(70.7)$ & $23(6.5)$ & $<0.001$ \\
\hline
\end{tabular}

*Means the number of variables is less than the total $\mathrm{N}=394$ because of missing data due to unperformed study tests. ${ }^{*}$ CXR was read by physician and reported as 'abnormal and suggestive of TB'.

Abbreviations: CXR chest X-ray, LAM Lipoarabinomannan, ART Antiretroviral therapy, IQR Inter-quartile range. 
Table 2 Multivariate analysis for predictors of mycobacteremia in sputum smear-negative HIV-infected presumptive TB patients*

\begin{tabular}{|c|c|c|c|c|}
\hline \multirow{3}{*}{ Characteristics } & \multicolumn{2}{|l|}{ Unadjusted OR } & \multicolumn{2}{|l|}{ Adjusted OR } \\
\hline & & $P$ value & & $P$ value \\
\hline & \multicolumn{2}{|l|}{$(95 \% \mathrm{Cl})$} & \multicolumn{2}{|l|}{$(95 \% \mathrm{Cl})$} \\
\hline Age (years) & $1.1(0.9-1.3)$ & 0.260 & - & - \\
\hline Hospitalization & $3.7(1.4-9.6)$ & 0.005 & - & - \\
\hline Male gender & $2.8(1.4-5.4)$ & 0.002 & $3.4(1.4-7.9)$ & 0.005 \\
\hline Not on ART & $2.0(1.0-4.2)$ & 0.07 & $1.5(0.5-4.0)$ & 0.128 \\
\hline CD4 $<100$ & 7.5(3.1-18.2) & $<0.001$ & $3.1(1.1-8.6)$ & 0.030 \\
\hline${ }^{* *}$ CXR abnormal & $1.2(0.6-2.5)$ & 0.631 & $0.8(0.3-2.0)$ & 0.637 \\
\hline Positive urine TB LAM test & $34.6(15.6-76.5)$ & $<0.001$ & $15.3(5.7-41.1)$ & $<0.001$ \\
\hline
\end{tabular}

${ }^{*} \mathrm{~N}=361$ with complete records. The model adjusted for gender, CD4 cell count, CXR findings, ART therapy, and positive lateral flow urine TB LAM test. ${ }^{* *}$ CXR read by physician and reported as 'abnormal and suggestive of TB'.

Abbreviations: CXR chest X-ray, LAM Lipoarabinomannan, ART Antiretroviral therapy, OR Odds ratio, Cl Confidence intervals.

did not reach statistical significance (Log rank test, $\mathrm{p}=$ 0.065) (Figure 2). Nearly $66.7 \%$ of all the deaths that occurred among patients with mycobacteremia occurred within the first three months of enrolment. Mortality outcome among the study participants when further stratified by urine TB LAM test results is shown in Table 3.

Of the 34 patients with mycobacteremia that had follow-up information available, 64.7\% (22/34) had been initiated on TB treatment at the time the MTB blood culture results were positive (Table 3 ). Compared to patients with MTB bacteremia who did not receive TB treatment, the proportion of patients who died by 12 months was significantly lower in the mycobacteremia patients who received TB treatment $[22.7 \%, 5 / 22$ vs. $58.3 \%(7 / 12) \mathrm{p}=0.0473]$.

\section{Discussion}

In this high prevalence TB/HIV setting, nearly one in every ten HIV-infected smear-negative presumptive TB patients are likely to have MTB bacteremia. Advanced
HIV (CD4 cell count $<100$ cells $/ \mathrm{mm}^{3}$ ), male gender and positive urine TB LAM test predicted presence of MTB bacteremia among smear-negative clinically suspected TB patients. Similar to prior reports from SSA $[12,18-20,28]$, we found a high prevalence of mycobacteremia among TB/HIV co-infected patients whose TB was not diagnosed by routine smear microscopy. Although sputum TB culture could identify $75 \%$ of the patients with mycobacteremia in our study, this test is not routinely performed in most high TB/HIV prevalence resource-limited settings [22]. CXR, another commonly available TB diagnostic method, was reported as abnormal and suggestive of TB in only $62 \%$ of the patients with mycobacteremia. Our findings confirm earlier reports that a significant proportion of mycobacteremic patients cannot be diagnosed using routinely available radiological and laboratory tests [12,18-20,28].

We found a trend towards increased mortality in HIVinfected smear-negative patients who had mycobacteremia

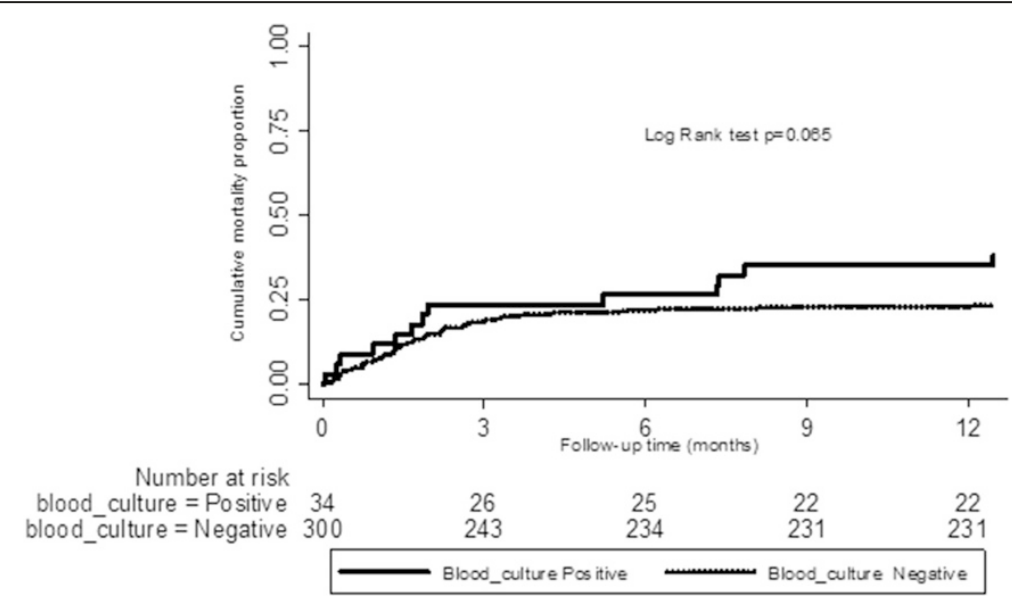

Figure 2 Kaplan-Meier curve comparing mortality between participants with and without MTB bacteremia. 
Table 3 Summary of 12-month follow-up outcomes for HIV-infected smear-negative participants stratified by TB blood culture status

\begin{tabular}{lll}
\hline & $\begin{array}{l}\text { MTB blood culture positive (N=41), } \\
\text { proportion (\%) }\end{array}$ & $\begin{array}{l}\text { Blood culture negative (N= 353), } \\
\text { proportion (\%) }\end{array}$ \\
\hline Lost to follow-up & $7 / 41(17)$ & $53 / 353(15)$ \\
12 months follow-up data available & $34 / 41(83 \%)$ & $300 / 353(85)$ \\
*Overall mortality & $12 / 34(35.3)$ & $70 / 300(23.3)$ \\
Mortality by urine LAM status** & & $7 / 19(36.8)$ \\
$\quad$ *Urine TB LAM positive and died & $8 / 23(34.8)$ & $62 / 280(22.1)$ \\
*Urine TB LAM negative and died & $4 / 11(36.4)$ & $58 / 300(19.3)$ \\
*TB treatment initiated & $22 / 34(64.7)$ & $242 / 300(80.7)$ \\
*No TB treatment & $12 / 34(35.3)$ & $12 / 58(20.7)$ \\
*TB treatment initiated and died & $5 / 22(22.7)$ & $58 / 242(24.0)$ \\
*Not initiated on TB treatment and died & $7 / 12(58.3)$ & \\
\hline
\end{tabular}

*Proportions were calculated among participants that had follow-up data available.

**One patient did not have a LAM test performed.

when compared to those without mycobacteremia even with this small sample size. Nearly two-thirds of the blood culture positive patients died within the first three months of enrolment. Blood culture services are still limited in SSA and it is unlikely that these services will become widely available in many $\mathrm{TB} / \mathrm{HIV}$ prevalent resourcelimited settings. We found that immunologically advanced HIV (CD4 cell count $<100$ cells $/ \mathrm{mm}^{3}$ ) and male gender were independently associated with mycobacteremia among HIV-infected sputum smear-negative patients, consistent with previous reports [15,29]. The association between male gender and mycobacteremia may be explained in part by the poorer health seeking behavior in men compared to women in SSA [30]. Many more men present with advanced immunosuppression and opportunistic infections compared to women.

Urine TB LAM was an independent predictor for mycobacteremia among HIV-infected smear negativeclinically suspected TB patients. The lateral-flow urine TB LAM test yields rapid results in 25 minutes and is very easy to perform. Therefore, urine TB LAM test could be used to predict mycobacteremia in smearnegative HIV-infected presumptive TB patients within a few hours after presentation. Early identification and treatment of smear-negative patients who are likely to have mycobacteremia could reduce attributable mortality. Further, similar to an earlier South African report [31], over two thirds of the participants with mycobacteremia had a positive urine TB LAM test. Our findings provide additional evidence that urine TB LAM test may be beneficial in patients with disseminated TB. A correlation between LAM positivity and patient outcomes has been previously published [32].

Our study had limitations. First, we did not perform autopsy studies for participants who died during the study and we were unable to establish definitively the actual cause of death in the study population. Secondly, we may not have found statistically significant differences in some of the comparisons made between the mycobacteremia and non-mycobacteremia patients due to the small sample size, particularly in the mycobacteremia group. Thirdly, mycobacteremia was assessed by inoculation of $3 \mathrm{ml}$ of blood into MYCO/F LYTIC cultures on a single occasion and thus mycobacteremia in the population could have been underestimated. The yield of MTB blood cultures may have been increased substantially by testing larger volumes of blood and by sampling on more than one occasion. Lastly, we could not determine whether blood culture results had additional benefits for clinical decision-making regarding the management of the sputum smear-negative participants and we could not ascertain the basis for the clinical decisions made by the attending physicians.

Despite these limitations, we report findings from both inpatient and outpatient populations that were recruited and underwent rigorous, protocol-specified laboratory testing. Our study is among only a few studies in the field of MTB bacteremia and highlights how clinical correlates and urine TB LAM test can be utilized in clinical management of smear-negative HIV-infected patients with mycobacteremia in a resource- limited setting. We believe that our findings are reliable and generalizable to most high TB/HIV prevalence resource-limited settings.

\section{Conclusion}

Mycobacteremia occurred in $10 \%$ of smear-negative patients and was associated with higher mortality compared with smear-negative patients without mycobacteremia. Advanced HIV disease $\left(\mathrm{CD} 4<100\right.$ cells $\left./ \mathrm{mm}^{3}\right)$, male gender and positive lateral flow urine TB LAM test predicted mycobacteremia in HIV-infected smear- 
negative presumptive TB patients in this high prevalence TB/HIV setting.

\section{Funding}

$\mathrm{LN}$ is a PhD candidate supported by by Medical Education for Equitable Services to All Ugandans a Medical Education Partnership Initiative grant number 5R24TW008886 from the Office of Global AIDS Coordinator and the U. S. Department of Health and Human Services, Health Resources and Services Administration and National Institutes of Health. The study project was supported by the Division of Microbiology and Infectious Diseases, National Institute of Allergy and Infectious Diseases, National Institutes of Health, Department of Health and Human Services (contract number HHSN2722000900050C to "TB Clinical Diagnostics Research Consortium"). Additional support was to YCM by the Johns Hopkins University Center for AIDS Research (Grant Number 1P30AI094189 from the National Institute of Allergy and Infectious Diseases). Its contents are solely the responsibility of the authors and do not necessarily represent the official views of the government.

\section{Competing interests}

The authors declare that they have no competing interests.

\section{Authors' contributions}

LN, WS, DN, MLJ, HMK, YCM conceived the idea; LN, YCM, JJE, SED obtained funding, LN, WS, DA, MH, MS acquired the Data; LN, WS, BK, HMK, MS, YCM performed the analysis and interpretation, LN primarily drafted the article. All authors contributed to revising this manuscript and gave final approval.

\section{Acknowledgements}

The authors would like to thank the research team at the Infectious Diseases Institute of College of Health Sciences, Makerere University Kampala, particularly Gloria Lubega, John Mark Bwanika, Olive Mbabazi, Allan Buzibye, Francis Kakooza, Joseph Sempa and the staff of Mycobacteriology Laboratory of Makerere University Kampala, for their important contributions to the implementation of this study. The authors gratefully acknowledge the study participants for their willingness to participate in the study.

\section{Author details}

${ }^{1}$ Infectious Diseases Institute, Makerere University College of Health Sciences, Kampala, Uganda. ${ }^{2}$ Makerere University College of Heath Sciences, Kampala, Uganda. ${ }^{3}$ Academic Medical Centre, University of Amsterdam, Amsterdam, The Netherlands. ${ }^{4} J o h n s$ Hopkins University School of Medicine, Baltimore, MD, USA. ${ }^{5}$ Boston Medical Center, Boston University School of Medicine, Boston, MA, USA.

Received: 22 January 2015 Accepted: 9 February 2015

Published online: 15 February 2015

\section{References}

1. Frieden TR, Sterling TR, Munsiff SS, Watt CJ, Dye C. Tuberculosis. Lancet. 2003:362(9387):887-99

2. Harries AD, Hargreaves NJ, Kemp J, Jindani A, Enarson DA, Maher D, et al. Deaths from tuberculosis in sub-Saharan African countries with a high prevalence of HIV-1. Lancet. 2001;357(9267):1519-23.

3. Corbett EL, Watt CJ, Walker N, Maher D, Williams BG, Raviglione MC, et al. The growing burden of tuberculosis: global trends and interactions with the HIV epidemic. Arch Intern Med. 2003;163(9):1009-21.

4. Getahun H, Gunneberg C, Granich R, Nunn P. HIV infection-associated tuberculosis: the epidemiology and the response. Clin Infect Dis. 2010;50 Suppl 3:S201-7.
5. Colebunders R, Bastian I. A review of the diagnosis and treatment of smearnegative pulmonary tuberculosis. Int J Tuberc Lung Dis. 2000;4(2):97-107.

6. Apers L, Wijarajah C, Mutsvangwa J, Chigara N, Mason P, van der Stuyft P. Accuracy of routine diagnosis of pulmonary tuberculosis in an area of high HIV prevalence. Int J Tuberc Lung Dis. 2004;8(8):945-51.

7. Nunn PP, Elliott AM, McAdam KP. Tropical respiratory medicine. 2. Impact of human immunodeficiency virus on tuberculosis in developing countries. Thorax. 1994;49(5):511-8.

8. Elliott AM, Namaambo K, Allen BW, Luo N, Hayes RJ, Pobee JO, et al. Negative sputum smear results in HIV-positive patients with pulmonary tuberculosis in Lusaka. Zambia Tuber Lung Dis. 1993;74(3):191-4.

9. Hargreaves NJ, Kadzakumanja O, Whitty CJ, Salaniponi FM, Harries AD, Squire SB. 'Smear-negative' pulmonary tuberculosis in a DOTS programme: poor outcomes in an area of high HIV seroprevalence. Int J Tuberc Lung Dis. 2001;5(9):847-54.

10. Henegar C, Behets F, Vanden Driessche K, Tabala M, Bahati E, Bola V, et al. Mortality among tuberculosis patients in the Democratic Republic of Congo. Int J Tuberc Lung Dis. 2012;16(9):1199-204.

11. Macpherson $P$, Dimairo M, Bandason T, Zezai A, Munyati SS, Butterworth AE, et al. Risk factors for mortality in smear-negative tuberculosis suspects: a cohort study in Harare. Zimbabwe Int J Tuberc Lung Dis. 2011;15(10):1390-6.

12. Ssali FN, Kamya MR, Wabwire-Mangen F, Kasasa S, Joloba M, Williams D, et al. A prospective study of community-acquired bloodstream infections among febrile adults admitted to Mulago Hospital in Kampala, Uganda. J Acquir Immune Defic Syndr Hum Retrovirol. 1998;19(5):484-9.

13. Archibald LK, McDonald LC, Nwanyanwu O, Kazembe P, Dobbie H, Tokars J, et al. A hospital-based prevalence survey of bloodstream infections in febrile patients in Malawi: implications for diagnosis and therapy. J Infect Dis. 2000;181(4):1414-20.

14. Crump JA, Ramadhani HO, Morrissey AB, Saganda W, Mwako MS, Yang LY, et al. Bacteremic disseminated tuberculosis in sub-saharan Africa: a prospective cohort study. Clin Infect Dis. 2012;55(2):242-50.

15. Reddy EA, Shaw AV, Crump JA. Community-acquired bloodstream infections in Africa: a systematic review and meta-analysis. Lancet Infect Dis. 2010;10(6):417-32.

16. Jacob ST, Pavlinac PB, Nakiyingi L, Banura P, Baeten JM, Morgan K, et al. Mycobacterium tuberculosis bacteremia in a cohort of hiv-infected patients hospitalized with severe sepsis in uganda-high frequency, low clinical suspicion [corrected] and derivation of a clinical prediction score. PLoS One. 2013;8(8):e70305.

17. Kethireddy S, Light RB, Mirzanejad Y, Maki D, Arabi Y, Lapinsky S, et al. Mycobacterium tuberculosis Septic Shock. Chest. 2013;144(2):474-82.

18. McDonald LC, Archibald LK, Rheanpumikankit S, Tansuphaswadikul S, Eampokalap B, Nwanyanawu O, et al. Unrecognised Mycobacterium tuberculosis bacteraemia among hospital inpatients in less developed countries. Lancet. 1999;354(9185):1159-63.

19. Harries AD. Unrecognised Mycobacterium tuberculosis. Lancet. 2000;355 (9198): 142. author reply 142-143.

20. Grinsztejn B, Fandinho FC, Veloso VG, Joao EC, Lourenco MC, Nogueira SA, et al. Mycobacteremia in patients with the acquired immunodeficiency syndrome. Arch Intern Med. 1997;157(20):2359-63.

21. Cox JA, Lukande RL, Nelson AM, Mayanja-Kizza H, Colebunders R, Van Marck E, et al. An autopsy study describing causes of death and comparing clinico-pathological findings among hospitalized patients in Kampala. Uganda PLoS One. 2012;7(3):e33685.

22. Archibald LK, Reller LB. Clinical microbiology in developing countries. Emerg Infect Dis. 2001;7(2):302-5.

23. Crump JA, Morrissey AB, Ramadhani HO, Njau BN, Maro VP, Reller LB. Controlled comparison of BacT/Alert MB system, manual Myco/F lytic procedure, and isolator 10 system for diagnosis of Mycobacterium tuberculosis Bacteremia. J Clin Microbiol. 2011;49(8):3054-7.

24. Hanscheid T, Monteiro C, Cristino JM, Lito LM, Salgado MJ. Growth of Mycobacterium tuberculosis in conventional BacT/ALERT FA blood culture bottles allows reliable diagnosis of Mycobacteremia. J Clin Microbiol. 2005;43(2):890-1.

25. Nakiyingi L, Moodley VM, Manabe YC, Nicol MP, Holshouser M, Armstrong DT, et al. Diagnostic accuracy of a rapid urine lipoarabinomannan test for tuberculosis in HIV-infected adults. J Acquir Immune Defic Syndr. 2014;66(3):270-9.

26. Infectious Diseases Institute Annual report; Infectious Diseases Institute, College of Health sciences Makerere University. http://idi.mak.ac.ug. In.; 2012. 
27. Uganda Ministry of Health Manual of the National Tuberculosis and Leprosy Programme, 2nd Edition, 2010. http://www.who.int/hiv/pub/guidelines/ uganda_tb.pdf, downloaded on 2nd January 2014.

28. Cox JA, Lukande RL, Lucas S, Nelson AM, Van Marck E, Colebunders R. Autopsy causes of death in HIV-positive individuals in sub-Saharan Africa and correlation with clinical diagnoses. AIDS Rev. 2010;12(4):183-94.

29. Lewis DK, Peters RP, Schijffelen MJ, Joaki GR, Walsh AL, Kublin JG, et al. Clinical indicators of mycobacteraemia in adults admitted to hospital in Blantyre, Malawi. Int J Tuberc Lung Dis. 2002;6(12):1067-74.

30. Peacock D, Redpath J, Weston M, Evans K, Daub A, Greig A, et al. Literature review on men, gender, health and HIV and AIDS in South Africa. Sonke Gender Justice Network 2008. http://menengage.org/wpcontent/uploads/ 2014/06/Male_attitudes_to_HIVAIDS_and_health_issues_in_South_Africa. pdf, downloaded on 7th January 2015

31. Shah M, Martinson NA, Chaisson RE, Martin DJ, Variava E, Dorman SE. Quantitative analysis of a urine-based assay for detection of lipoarabinomannan in patients with tuberculosis. J Clin Microbiol. 2010;48(8):2972-4.

32. Manabe YC, Nonyane BA, Nakiyingi L, Mbabazi O, Lubega G, Shah M, et al. Point-of-care lateral flow assays for tuberculosis and cryptococcal antigenuria predict death in HIV infected adults in Uganda. PLoS One. 2014;9(7):e101459.

\section{Submit your next manuscript to BioMed Central and take full advantage of:}

- Convenient online submission

- Thorough peer review

- No space constraints or color figure charges

- Immediate publication on acceptance

- Inclusion in PubMed, CAS, Scopus and Google Scholar

- Research which is freely available for redistribution 